\title{
Simulation and refraction event of complex hyperbolic type solitary wave in plasma and obtical fiber for the perturbed Chen-Lee-Liu equation
}

\section{Asıf Yokuş}

Firat Universitesi

Hülya Durur ( $\nabla$ hulyadurur@ardahan.edu.tr)

Ardahan Universitesi https://orcid.org/0000-0002-9297-6873

Serbay Duran

Adıyaman Üniversitesi: Adiyaman Universitesi

\section{Research Article}

Keywords: The modified $1 / G^{\prime}$-expansion method, modified Kudryashov method, the perturbed Chen-LeeLiu equation, wave solution

Posted Date: March 17th, 2021

DOl: https://doi.org/10.21203/rs.3.rs-300529/v1

License: (c) (i) This work is licensed under a Creative Commons Attribution 4.0 International License. Read Full License

Version of Record: A version of this preprint was published at Optical and Quantum Electronics on July 14th, 2021. See the published version at https://doi.org/10.1007/s11082-021-03036-1. 


\title{
Simulation and refraction event of complex hyperbolic type solitary wave in plasma and obtical fiber for the perturbed Chen-Lee-Liu equation
}

\author{
Asıf Yokuş ${ }^{1}$, Hülya Durur ${ }^{2 *}$, Serbay Duran ${ }^{3}$ \\ ${ }^{1}$ Department of Actuary, Faculty of Science, Firat University, Elazig, 23100, Turkey \\ asfyokus@yahoo.com \\ ${ }^{2 *}$ Department of Computer Engineering, Faculty of Engineering, Ardahan University, Ardahan,75000, Turkey \\ hulyadurur@ardahan.edu.tr, \\ ${ }^{3}$ Department of Mathematics and Science Education, Faculty of Education, Adlyaman University, \\ Adlyaman, 02040, Turkey \\ sduran@adiyaman.edu.tr
}

\begin{abstract}
In this presented article, modified $1 / G^{\prime}$-expansion and modified Kudryashov methods are applied to generate traveling wave solutions of perturbed Chen-Lee-Liu (CLL) equation. The similar and different aspects of the solutions produced by both analytical methods are discussed in the results and discussion section. By giving special values to the constants in the solutions obtained by analytical methods, 2D, 3D and contour graphics representing the shape of the standing wave at any time are presented. Additionally, the advantages and disadvantages of the two analytical methods are discussed and presented in the results and discussion section. Also, a solitary wave is produced by giving special values to the parameters in the hyperbolic type complex traveling wave solution. Simulations are created for different values of the frequency and velocity propagation parameters of the solitary wave. The values of these parameters are calculated for the breakage event physically. A computer package program is used for operations such as solving complex operations, drawing graphics and systems of algebraic equations.
\end{abstract}

Keywords: The modified $1 / G^{\prime}$-expansion method, modified Kudryashov method, the perturbed Chen-Lee-Liu equation, wave solution.

\section{Introduction}

In recent years, major advances have been observed in studies on analytical solutions of nonlinear partial differential equations (NLPDEs). These equations are mathematical models of physical phenomena that occur in interdisciplinary fields of mathematics. Analytical solutions of these equations provide information about the character of the physical event that describes the equation. Therefore, scientists work hard to obtain the solutions of these equations. Thanks to these studies of scientists, many analytical methods have been developed. Solving NLPDEs are more difficult and time consuming than solving ordinary differential equations (ODEs). To overcome these difficulties, NLPDEs are converted into ODEs. Scientists usually use a variable transformation to do this transformation. In many of these analytical methods are used linear or nonlinear ordinary differential equations as an arbitrary equation. Analytical solutions of the investigated problem are obtained by making use of different types solution functions of these 
arbitrary equations. When some researchers use the riccati differential equation or the second order ordinary differential equation as the arbitrary equation, they obtain different trigonometric, hyperbolic and rational solution functions of the problem under consideration. Different analytical methods have been developed according to the selection of these arbitrary equations. For example, some methods have been developed by using different types of $G^{\prime \prime}+\lambda G^{\prime}+\mu G=0$. Some of these methods: $\left(G^{\prime} / G\right)$-expansion method [1], $\left(G^{\prime} / G^{2}\right)$-expansion method [2], (1/G')-expansion method [3-7], $\left(m+G^{\prime} / G\right)$-expansion method [8], $\left(m+1 / G^{\prime}\right)$ expansion method [9], two variables $\left(G^{\prime} / G, 1 / G\right)$-expansion method [10-12]. Some scientists are presented different versions of these methods according to the selected solution function in the literature, such as extended, improved and generalized. In this sense, some methods are modified simple equation method [13], modified extended tanh-function method [14], modified hyperbolic-function expansion method [15], complex method [16], Haar wavelet method $[17,18]$, auxiliary equation method [19], sub equation method [20] and many more methods [21-31].

In this study, we consider the CLL equation that has many applications in plasma and optical fiber [32]. This equation was first published in 1979 [33]. Especially in the last few years, many studies have been conducted on the solutions of this equation. The mathematical model of the phenomena we encounter in plasma physics and fiber optics corresponds to a nonlinear differential equation such as the CLL equation. By applying analytical methods to a nonlinear differential equation, soliton solutions are obtained. When these soliton solutions are examined, special cases of these solutions are obtained, depending on the wave propagation parameter, height and density. We can classify these special cases as dark, bright and singular solitons [34]. Bright and dark solitons are related to the intensity of the wave. While bright solitons indicate an increase, dark solitons indicate a decrease in the intensity of the wave [35,36]. Singular solitons are a special case and are the result of a balance between the speed of the wave and its height [37].

In this article, authors established the soliton wave solutions of the following perturbed CLL equation that represents the propagation of an optical pulse in plasma and optical fiber:

$$
i q_{t}+a q_{x x}+i b|q|^{2} q_{x}=i\left[\lambda q_{x}+\theta\left(|q|^{2 m} q\right)_{x}+\sigma\left(|q|^{2 m}\right)_{x} q\right]
$$

the $x$ and $t$ coordinates are the wave propagation distance and retarded time, respectively. $q=q(x, t)$ is the complex wave function dependent on these independent coordinates. $\mathrm{a}$ is the velocity propagation parameter and $\mathrm{b}$ is the nonlinear dispersion parameter. $\sigma, \theta$ and $\lambda$ on the right side of the equation are coefficients of nonlinear dispersion, self-steeping for short 
pulses and the inter-modal dispersion, respectively. In addition, the nonlinearity parameter $m$ in the above equation defines the density for complex wave function [38].

\section{Description of the modified Kudryashov method}

Suppose you have a nonlinear evolution equation in the form below

$T\left(q, q_{t}, q_{x}, q_{x x}, \ldots\right)=0$,

where $T$ is a polynomial in $q(x, t)$ and its partial derivatives in which nonlinear terms and highest order derivatives. We give the basic steps of this method in the follow:

Step 1. Using the wave transformation

$q(x, t)=U(\xi) e^{i \varphi(x, t)}, \quad \xi=\eta(x-v t), \quad \varphi(x, t)=-\kappa x+w t+s$,

where $\kappa, w, v, s$ are constants and we can transform it the following ODE for $U(\xi)$ :

$S\left(U, U^{\prime}, U^{\prime \prime}, \ldots\right)=0$

Step 2. We assume that Eq. (4) has the formal solution

$U(\xi)=a_{0}+\sum_{i=1}^{n}\left(a_{i} Q(\xi)^{i}+a_{-i} Q(\xi)^{-i}\right)$,

where $a_{i}, a_{-i}, \quad i=\{1, \ldots, n\}$ are constants to be determined, such that $a_{n} \neq 0$ or $a_{-n} \neq 0$, and $Q(\xi)$ is the solution of the equation

$Q^{\prime}(\xi)=\left[Q^{2}(\xi)-Q(\xi)\right] \ln \tau$,

Eq. (6) has solutions

$Q(\xi)=\frac{1}{1 \pm \tau^{\xi}}$,

where $\tau>0, \tau \neq 1$ is a number.

Step 3. We identify the positive integer $n$ in Eq. (5) taking into account the homogeneous balance between the nonlinear terms and the highest order derivatives in Eq. (4).

Step 4. Substitute Eq. (5) with Eq. (6) into Eq. (4), we compute all the required derivatives $U^{\prime}, U^{\prime \prime}, \ldots$ of the function $U(\xi)$. As a result of this substitution, we get a polynomial of $Q^{j}(\xi),(j=0,1,2, \ldots)$. In this polynomial, we add all the terms of the same powers of $Q^{j}(\xi)$ and equal them to zero, we get a system of algebraic equations that can be solved by computer package program to attain the unknown parameters $a_{i}, a_{-i}, \quad i=\{1, \ldots, n\}, k$ and $v$. As a result, we get exact solutions of the Eq. (2). 


\section{Description of the modified $1 / G^{\prime}$-expansion method}

Consider the form of NLPDEs,

$\diamond\left(q, \frac{\partial q}{\partial t}, \frac{\partial q}{\partial x}, \frac{\partial^{2} q}{\partial x^{2}}, \ldots\right)=0$

Let $q(x, t)=U(\xi) e^{i \varphi(x, t)}, \quad \xi=\eta(x-v t), \quad \varphi(x, t)=-\kappa x+w t+s$, here $v$ is the velocity of the wave and constant. We may transform it the following ODE for $U(\xi)$ :

$\theta\left(U, U^{\prime}, U^{\prime \prime}, \ldots\right)=0$.

The solution of Eq. (9) is supposed that with the form

$$
U(\xi)=a_{0}+\sum_{i=1}^{n}\left(a_{i}\left(\frac{1}{G^{\prime}}\right)^{i}+a_{-i}\left(\frac{1}{G^{\prime}}\right)^{-i}\right),
$$

where $a_{i}, a_{-i}, \quad i=\{1, \ldots, n\}$ are scalars, $G=G(\xi)$ ensures following second-order IODE

$G^{\prime \prime}+\lambda G^{\prime}+\gamma=0$,

here $\gamma$ and $\lambda$ are constants to be determined,

$$
\frac{1}{G^{\prime}(\xi)}=\frac{1}{-\frac{\gamma}{\lambda}+A \cosh [\xi \lambda]-A \sinh [\xi \lambda]} .
$$

The software for Eq. (12) in exponential form is as follows

$$
\frac{1}{G^{\prime}(\xi)}=\frac{1}{-\frac{\gamma}{\lambda}+A e^{-\xi \lambda}} .
$$

The wanted derivatives of Eq. (10) are calculated and written into Eq. (9), obtaining a polynomial with $\left(1 / G^{\prime}\right)$. Equating the coefficients of this polynomial to zero, an algebraic system of equations is created. The Equation is solved via the package program and the default Eq. (9) is put in its place in the solution function. Eventually, the solutions of Eq. (8) are found.

\section{Application of modified Kudryashov Method}

We consider Eq. (1). By using

$$
q(x, t)=U(\xi) e^{i \varphi(x, t)}, \quad \xi=\eta(x-v t),
$$

where the amplitude component is $U(\xi)$, with $v$ being the velocity, while phase component

$\varphi(x, t)=-\kappa x+w t+s$, 
where the parameters $s, w, \kappa$ sequentially correspond to the phase, wave number and frequency. Inserting Eq. (2) into Eq. (1), the real and imaginary equations are

$$
\begin{aligned}
& a \eta^{2} U^{\prime \prime}-\left(w+a \kappa^{2}+\kappa \lambda\right) U+b \kappa U^{3}-\kappa \theta U^{2 m+1}=0, \\
& -v-2 a \kappa-\lambda+b U^{2}-(2 m \theta+\theta+2 \sigma m) U^{2 m}=0,
\end{aligned}
$$

respectively. For use the balancing rule, Eqs.(15) and (16) are modified to

$$
a \eta^{2} U^{\prime \prime}-\left(w+a \kappa^{2}+\kappa \lambda\right) U+2 \kappa(\theta+\sigma) U^{3}=0,
$$

and

$v=-2 a \kappa-\lambda, \quad b=3 \theta+2 \sigma$,

By setting $m=1$. The profile of the optical solitons is recovered by aid of integrating the Eq. (7) while the velocity of the optical solitons is emerged from the Eq. (18). In Eq. (18), we get the balancing term $n=1$ and by considering in the Eq. (5),

$U(\xi)=a_{0}+a_{1} Q(\xi)+a_{2} \frac{1}{Q(\xi)}$,

if Eq. (19) is written in Eq. (17) and if necessary adjustments are made, the following systems of equations can be written:

Const : $-a k^{2} a_{0}-w a_{0}-k \lambda a_{0}-2 k \theta a_{0}^{3}-2 k \sigma a_{0}^{3}-a \eta^{2} \log [\tau]^{2} a_{2}-12 k \theta a_{0} a_{1} a_{2}-12 k \sigma a_{0} a_{1} a_{2}=0$,

$\frac{1}{Q[\xi]}:-a k^{2} a_{2}-w a_{2}-k \lambda a_{2}+a \eta^{2} \log [\tau]^{2} a_{2}-6 k \theta a_{0}^{2} a_{2}-6 k \sigma a_{0}^{2} a_{2}-6 k \theta a_{1} a_{2}^{2}-6 k \sigma a_{1} a_{2}^{2}=0$

$\frac{1}{Q[\xi]^{2}}:-6 k \theta a_{0} a_{2}^{2}-6 k \sigma a_{0} a_{2}^{2}=0$

$\frac{1}{Q[\xi]^{3}}:-2 k \theta a_{2}^{3}-2 k \sigma a_{2}^{3}=0$,

$Q[\xi]:-a k^{2} a_{1}-w a_{1}-k \lambda a_{1}+a \eta^{2} \log [\tau]^{2} a_{1}-6 k \theta a_{0}^{2} a_{1}-6 k \sigma a_{0}^{2} a_{1}-6 k \theta a_{1}^{2} a_{2}-6 k \sigma a_{1}^{2} a_{2}=0$,

$Q[\xi]^{2}:-3 a \eta^{2} \log [\tau]^{2} a_{1}-6 k \theta a_{0} a_{1}^{2}-6 k \sigma a_{0} a_{1}^{2}=0$,

$Q[\xi]^{3}: 2 a \eta^{2} \log [\tau]^{2} a_{1}-2 k \theta a_{1}^{3}-2 k \sigma a_{1}^{3}=0$.

$a_{0}, a_{1}, a_{2}$ and $a, k, \theta, \sigma, \lambda, \eta, w$ constants are obtained from Eq. (20) the system utilizing a software program.

\section{Case1: If}

$a_{1}=0, \quad a_{2}=-a_{0}, \quad w=-a k^{2}-k \lambda+a \eta^{2} \log [\tau]^{2}, \quad \sigma=-\theta, \quad v=-2 a k-\lambda, \quad b=3 \theta+2 \sigma ;$ 
replacing values (21) into (19), we get traveling wave soliton for Eq. (1)

$q_{1}(x, t)=\mathrm{e}^{\mathrm{i}\left(s-k x+t\left(-a k^{2}-k \lambda+a \eta^{2} \log [\tau]^{2}\right)\right)}\left(a_{0}-\left(1+\tau^{\eta(x-t(-2 a k-\lambda))}\right) a_{0}\right)$.

The solution presented with Eq. (22) is known as traveling wave solution in the literature.
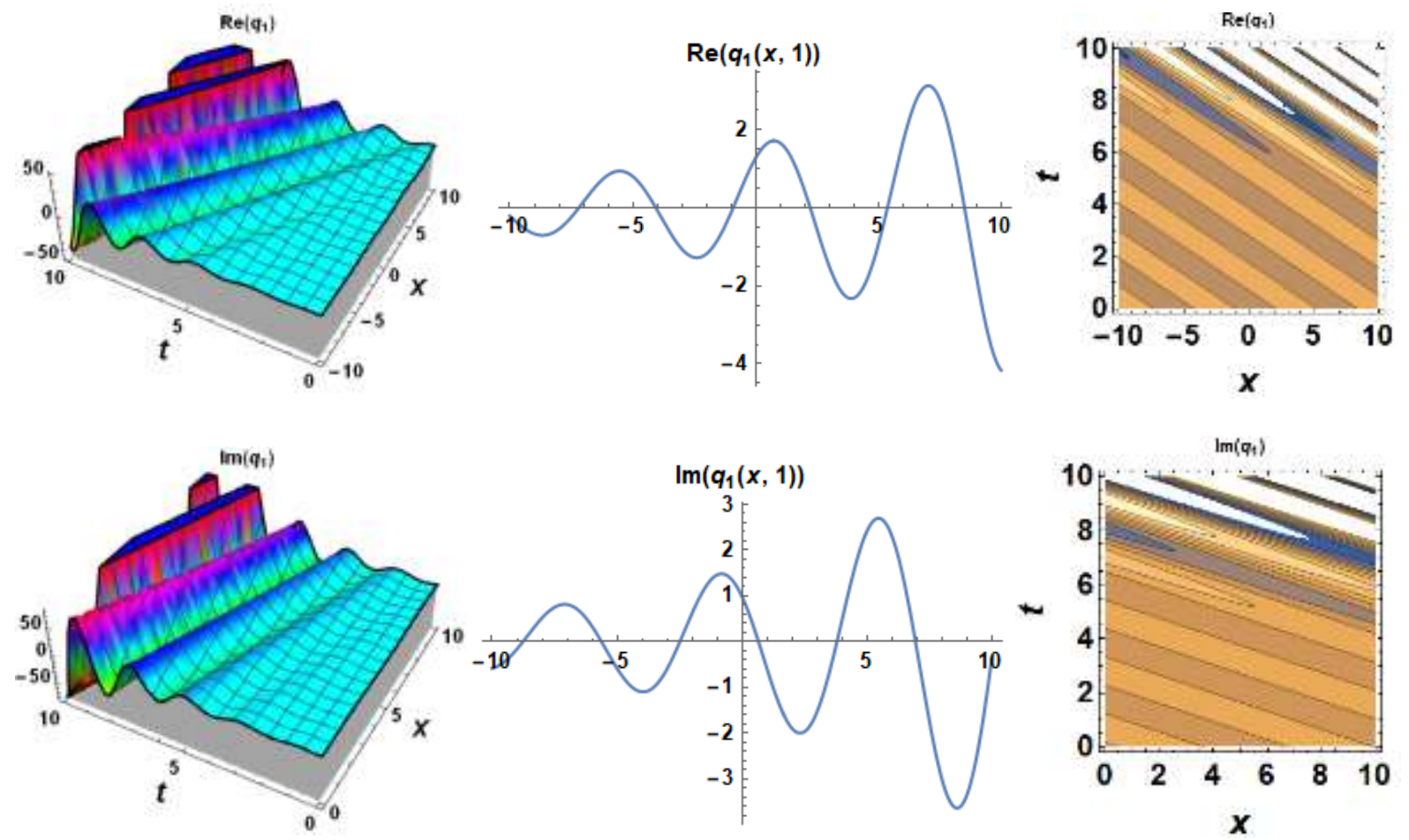

Fig.1. Real and imaginary parts of 3D, 2D and contour graphs for the Eq. (21) for $s=0.5, k=1, \eta=1, a=2, a_{0}=1, \tau=1.1, \lambda=1$.

\section{Case2: If}

$a_{2}=0, \quad w=-k \lambda, \quad \sigma=-\theta, \quad a=0, \quad v=-2 a k-\lambda, \quad b=3 \theta+2 \sigma$,

replacing values (23) into (19), we get traveling wave soliton for Eq. (1)

$q_{2}(x, t)=\mathrm{e}^{\mathrm{i}(s-k x-k t \lambda)}\left(a_{0}+\frac{a_{1}}{1+\tau^{\eta(x+t \lambda)}}\right)$.

The solution presented with Eq. (24) is known as traveling wave solution in the literature. 

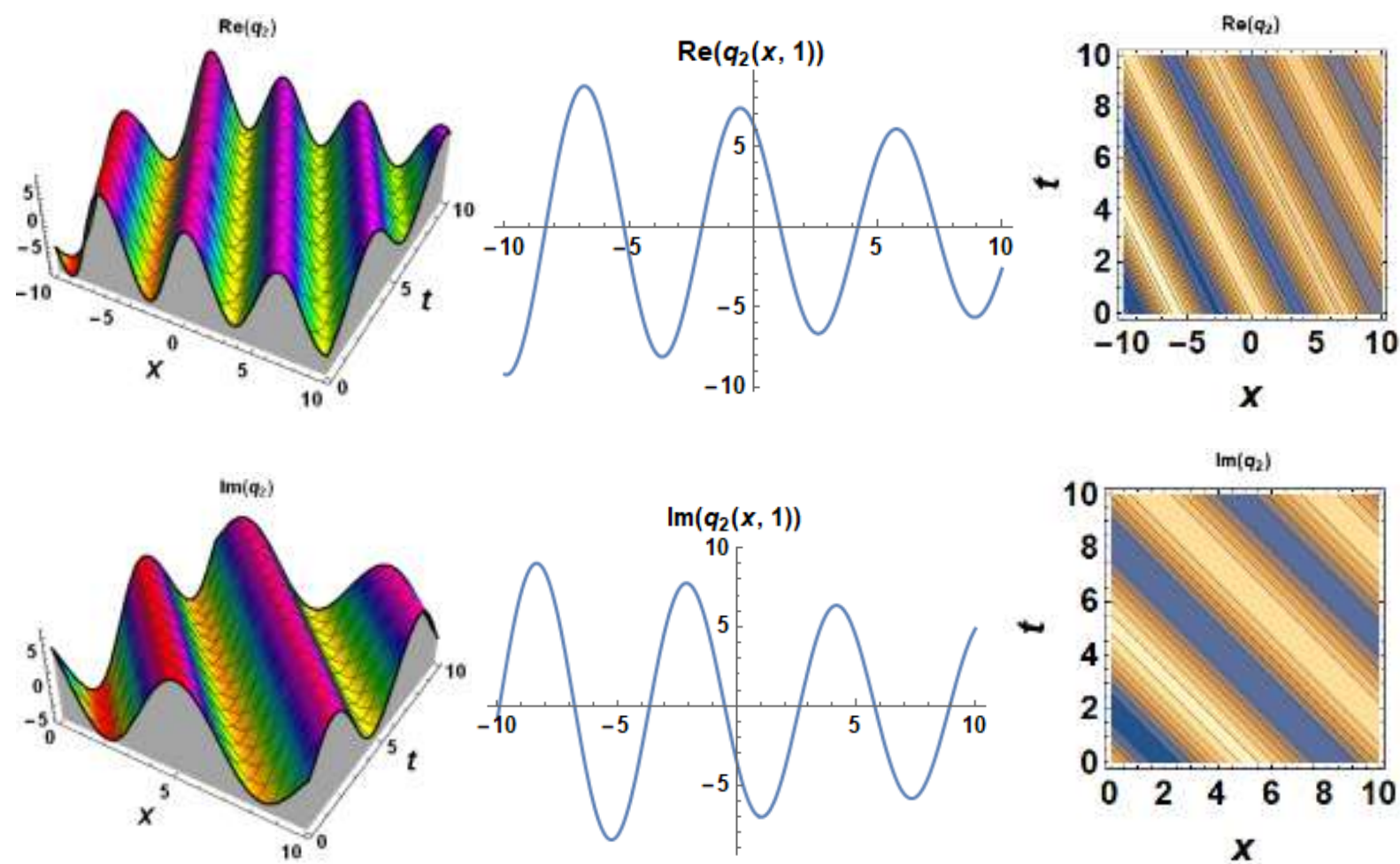

Fig.2. Real and imaginary parts of 3D, 2D and contour graphs of the Eq. (24) for $s=0.5, k=1, \eta=2, a_{1}=5, a_{0}=5, \tau=1.1, \lambda=1$.

\section{Application of the modified $\left(\frac{1}{G^{\prime}}\right)$-expansion method}

Let's take Eq. (17) here, $n=1$ is obtained according to the homogeneous balance principle. In Eq. (10), the following situation is obtained:

$U(\xi)=a_{0}+a_{1}\left(\frac{1}{G^{\prime}}\right)+a_{2} G^{\prime}$.

Substituting Eq. (25) in Eq. (17) and the coefficients of Eq. (1) are set to zero, the following systems of equations can be written: 
Const: $-a k^{2} a_{0}-w a_{0}-k \lambda a_{0}-2 k \theta a_{0}^{3}-2 k \sigma a_{0}^{3}+a \eta^{2} \mu \gamma a_{2}$

$-12 k \theta a_{0} a_{1} a_{2}-12 k \sigma a_{0} a_{1} a_{2}=0$,

$\frac{1}{G^{\prime}[\xi]}:-a k^{2} a_{1}-w a_{1}-k \lambda a_{1}+a \eta^{2} \gamma^{2} a_{1}-6 k \theta a_{0}^{2} a_{1}-6 k \sigma a_{0}^{2} a_{1}$

$-6 k \theta a_{1}^{2} a_{2}-6 k \sigma a_{1}^{2} a_{2}=0$,

$\frac{1}{G^{\prime}[\xi]^{2}}: \quad 3 a \eta^{2} \mu \gamma a_{1}-6 k \theta a_{0} a_{1}^{2}-6 k \sigma a_{0} a_{1}^{2}=0$,

$\frac{1}{G^{\prime}[\xi]^{3}}: \quad 2 a \eta^{2} \mu^{2} a_{1}-2 k \theta a_{1}^{3}-2 k \sigma a_{1}^{3}=0$,

$G^{\prime}[\xi]: \quad-a k^{2} a_{2}-w a_{2}-k \lambda a_{2}+a \eta^{2} \gamma^{2} a_{2}-6 k \theta a_{0}^{2} a_{2}-6 k \sigma a_{0}^{2} a_{2}$

$-6 k \theta a_{1} a_{2}^{2}-6 k \sigma a_{1} a_{2}^{2}=0$,

$G^{\prime}[\xi]^{2}: \quad-6 k \theta a_{0} a_{2}^{2}-6 k \sigma a_{0} a_{2}^{2}=0$,

$G^{\prime}[\xi]^{3}:-2 k \theta a_{2}^{3}-2 k \sigma a_{2}^{3}=0$.

$a_{0}, a_{1}, a_{2}$ and $c, v, \gamma, w, \kappa, \alpha, \lambda$ constants are obtained from Eq. (25) the system utilizing a software program.

\section{Case1. If}

$a_{1}=0, \quad w=-a k^{2}-k \lambda+a \eta^{2} \gamma^{2}, \quad \sigma=-\theta, \quad \mu=\frac{\gamma a_{0}}{a_{2}}, \quad v=-2 a k-\lambda, \quad b=3 \theta+2 \sigma$,

modifying values Eq. (27) in Eq. (25) and we have the complex hyperbolic type traveling wave solution for Eq. (1):

$q_{3}(x, t)=\mathrm{e}^{\mathrm{i}\left(s-k x+t\left(-a k^{2}-k \lambda+a \eta^{2} \gamma^{2}\right)\right)}\left(a_{0}+\left(A \mathrm{e}^{-\eta(x-t(-2 a k-\lambda)) \gamma}-\frac{a_{0}}{a_{2}}\right) a_{2}\right)$.

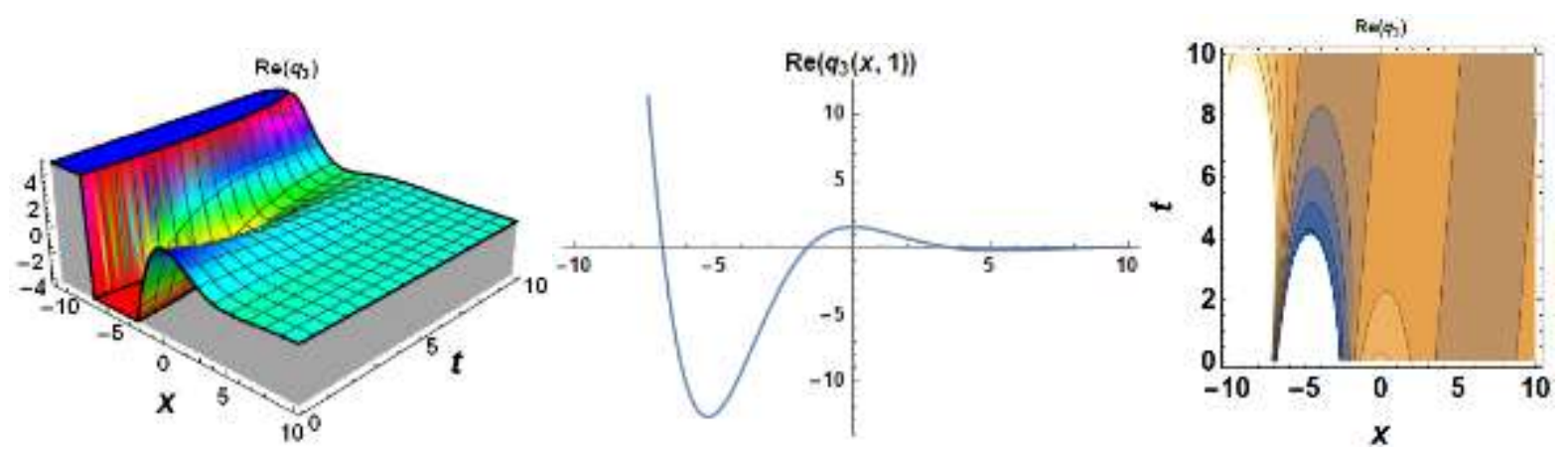



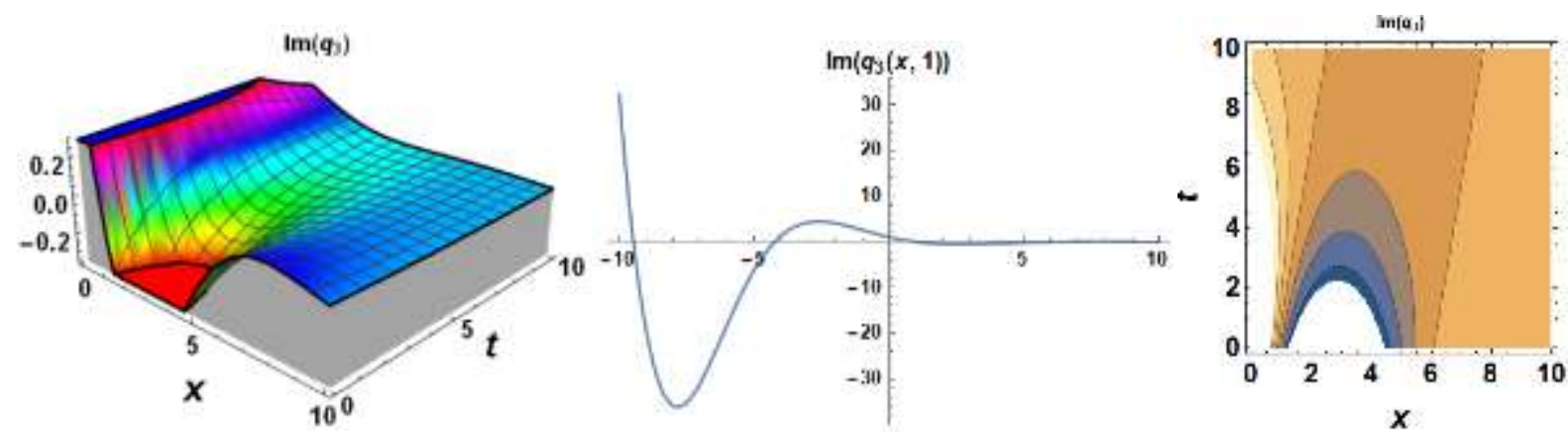

Fig.3: Real and imaginary parts of 3D, 2D and contour graphs of the Eq. (28) for $s=0.5, k=0.6, \eta=0.4, a=1, a_{0}=2, \gamma=1, \lambda=-0.5, a_{2}=1, A=2.5$.

The graphic presented in Fig. 3 is complex hyperbolic type traveling wave representing the stationary wave at any time.

\section{Case2: If}

$a_{0}=0, \quad w=-a k^{2}-k \lambda+a \eta^{2} \gamma^{2}, \quad \sigma=-\theta, \quad \mu=0, \quad v=-2 a k-\lambda, \quad b=3 \theta+2 \sigma$,

replacing values (29) into (25), we get complex hyperbolic traveling wave solution for Eq. (1)

$q_{4}(x, t)=\mathrm{e}^{\mathrm{i}\left(s-k x+t\left(-a k^{2}-k \lambda+a \eta^{2} \gamma^{2}\right)\right)}\left(\frac{\mathrm{e}^{\eta(x-t(-2 a k-\lambda)) \gamma} a_{1}}{A}+A \mathrm{e}^{-\eta(x-t(-2 a k-\lambda)) \gamma} a_{2}\right)$.
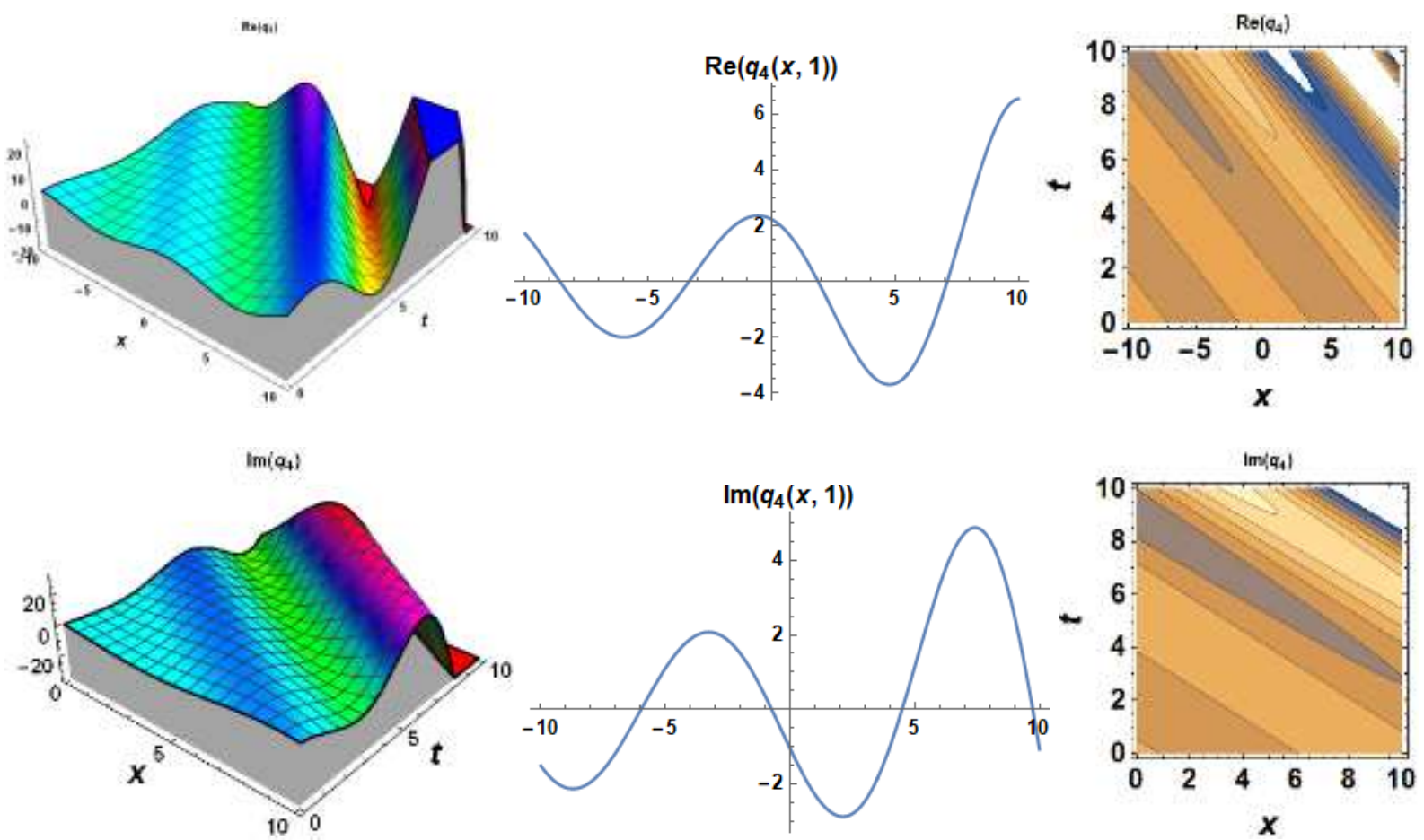
Fig.4: Real and imaginary parts of 3D, 2D and contour graphs for the Eq. (30) for $s=0.5, k=0.6, \eta=0.4, a=1, \gamma=-0.3, \lambda=1, a_{1}=1, a_{2}=1, A=1.5$.

The graphic presented in Fig. 4 is complex hyperbolic type traveling wave representing the stationary wave at any time.

\section{Case3: If}

$a_{1}=0, \quad w=-a k^{2}-k \lambda+a \eta^{2} \gamma^{2}, \quad \sigma=-\theta, \quad \mu=\frac{\gamma a_{0}}{a_{2}}, \quad v=-2 a k-\lambda, \quad b=3 \theta+2 \sigma$,

replacing values (30) into (25), we get complex hyperbolic traveling wave solution for Eq. (1)

$q_{5}(x, t)=\mathrm{e}^{\mathrm{i}\left(s-k x+t\left(-a k^{2}-k \lambda+a \eta^{2} \gamma^{2}\right)\right)}\left(a_{0}+\left(\begin{array}{l}A \cosh [\eta(x-t(-2 a k-\lambda)) \gamma] \\ -A \sinh [\eta(x-t(-2 a k-\lambda)) \gamma]-\frac{a_{0}}{a_{2}}\end{array}\right) a_{2}\right)$.
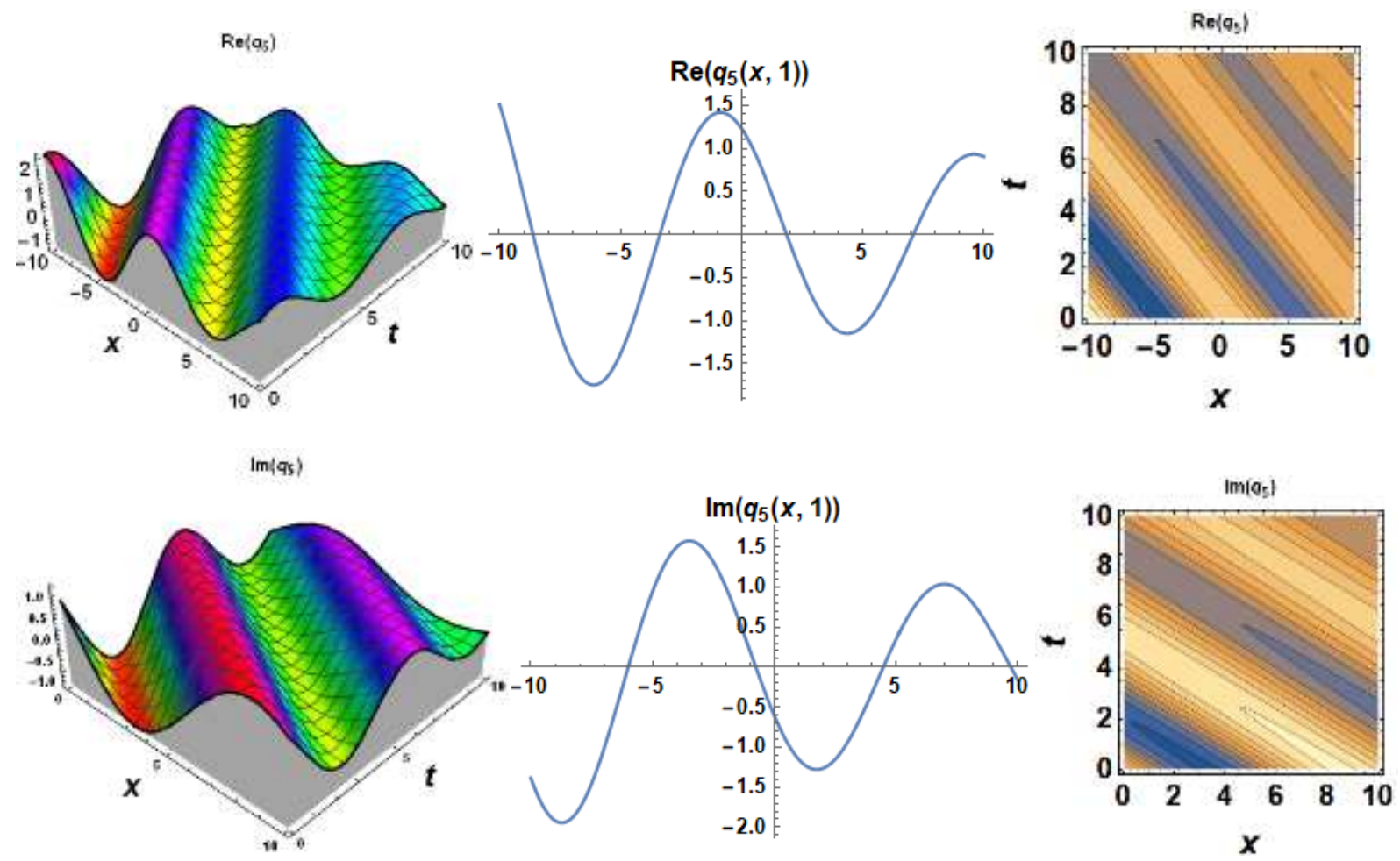

Fig.5: Real and imaginary of 3D, 2D and contour graphs of the Eq. (32) for $s=0.5, k=0.6, \eta=0.4, a=1, \gamma=0.1, \lambda=1, a_{0}=1, a_{2}=1, A=1.5$.

The graphic presented in Fig. 5 is complex hyperbolic type traveling wave representing the stationary wave at any time.

\section{Case4: If}

$a_{0}=0, \quad a_{2}=0, \quad w=-a k^{2}-k \lambda+a \eta^{2} \gamma^{2}, \quad \sigma=-\theta, \quad \mu=0, \quad v=-2 a k-\lambda, \quad b=3 \theta+2 \sigma$, 
replacing values (33) into (25), we get complex hyperbolic traveling wave solution for Eq. (1)

$$
q_{6}(x, t)=\frac{\mathrm{e}^{\mathrm{i}\left(s-k x+t\left(-a k^{2}-k \lambda+a \eta^{2} \gamma^{2}\right)\right)} a_{1}}{A \cosh [\eta(x-t(-2 a k-\lambda)) \gamma]-A \sinh [\eta(x-t(-2 a k-\lambda)) \gamma]} .
$$
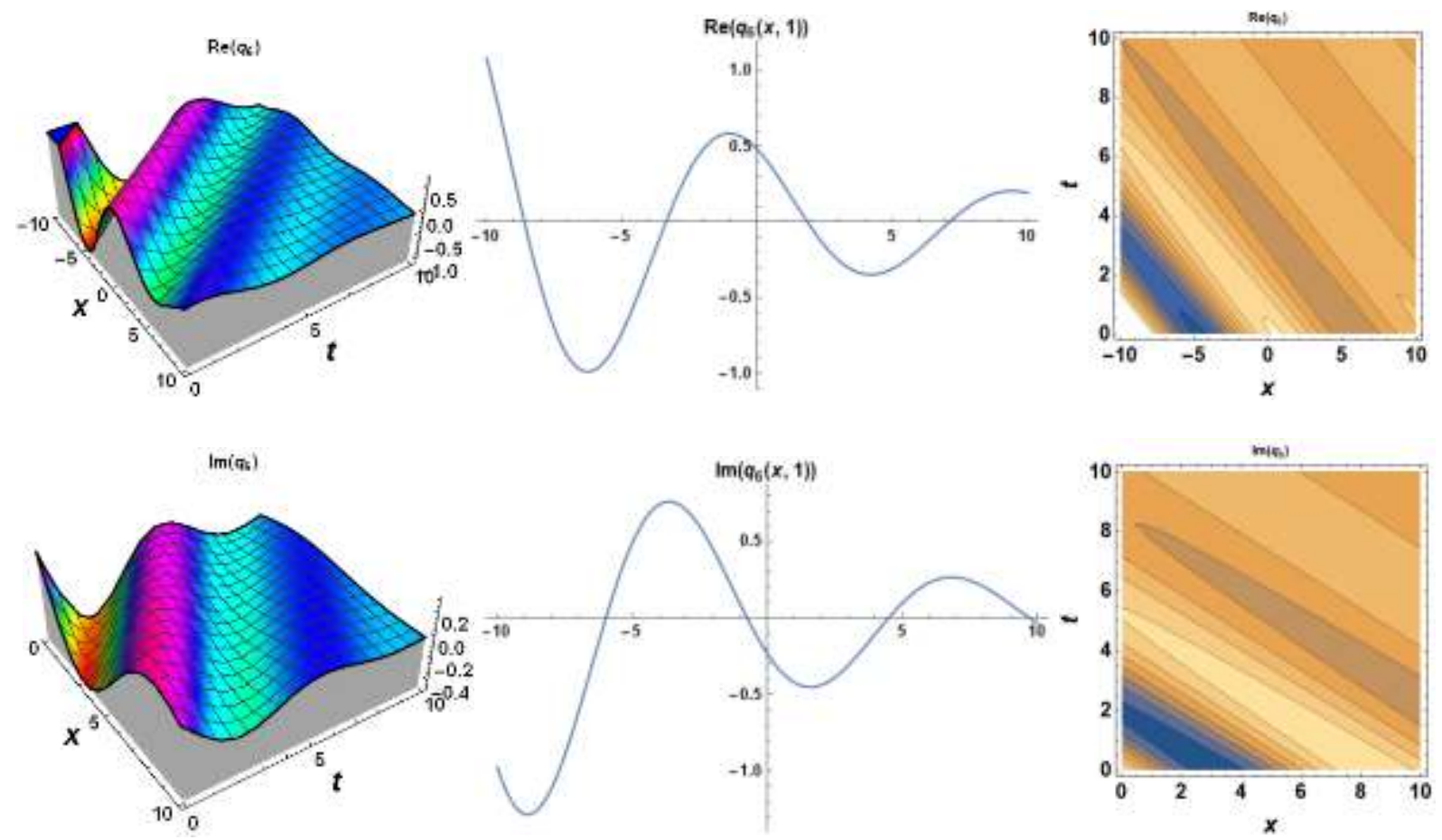

Fig.6: Real and imaginary of 3D, 2D and contour graphs of the Eq. (34) for $s=0.5, k=0.6, \eta=0.5, a=1, \gamma=-0.2, \lambda=1, a_{1}=1, A=1.5$.

The graphic presented in Fig. 6 is complex hyperbolic type traveling wave representing the stationary wave at any time.

\section{Results and Discussions}

Traveling wave solutions have an important place in Soliton's theory. Many researchers have focused on the solutions of partial differential equations. In this study, we have produced the traveling wave solutions by successfully applying the modified $1 / G^{\prime}$-expansion and modified Kudryashov methods of the nonlinear perturbed CLL equation. A modified version of both analytical methods were applied for the first time. At the end of this application, we found that both analytical methods are reliable, applicable, effective and useful. There are similarities and differences between the solutions produced by both analytical methods. While the base equation used in the modified $1 / G^{\prime}$-expansion is Eq.(11), it is Eq. (6) in the modified Kudryashov method. Although the base equations used are different, there are also similarities in the solutions. When the solution format given by (12.a) Eq. (6) in the modified $1 / G^{\prime}$ - expansion method is compared with the solution format given by the Eq. (7) of the modified Kudryashov method, having the constants in the Eq. (12.a) carries a more general solution. However, we can conclude that base $a$ in Eq. (7) is a free parameter and it is a more general solution compared 
to the modified $1 / G^{\prime}$ - expansion method. Although the solutions produced by both analytical methods are similar, they add different interpretations in terms of physical meaning. This is also a wealth of mathematics. If we discuss the advantages and disadvantages of the methods, we can say that both methods are at the same level in terms of transaction complexity. Qualitatively, both methods produce traveling wave solutions and quantitatively the number of solutions produced by the modified $1 / G^{\prime}$-expansion method is higher. The solutions presented in this study are hyperbolic type complex traveling wave solutions. The term that affects the Eq. (32) directly in the real part and indirectly in the imaginary part is the coefficient " $a$ ". The term that directly affects both the real and imaginary parts of the solution presented with Eq. (32) is the term $\eta$. Let's examine the effect of changes in both $\eta$ and coefficient " $a$ " on wave behavior. First, let's explain the behavior changes of the coefficient " $a$ " representing the wave propagation speed in the solitary wave solution with the help of 3-dimensional simulation. In Fig. 7, the behavior of the wave is presented for different values of the wave propagation velocity coefficient " $a$ " in Eq. (1). In addition, the velocity value that causes the occurrence of the physical phenomenon called refraction in wave behavior can be observed in the simulation below.
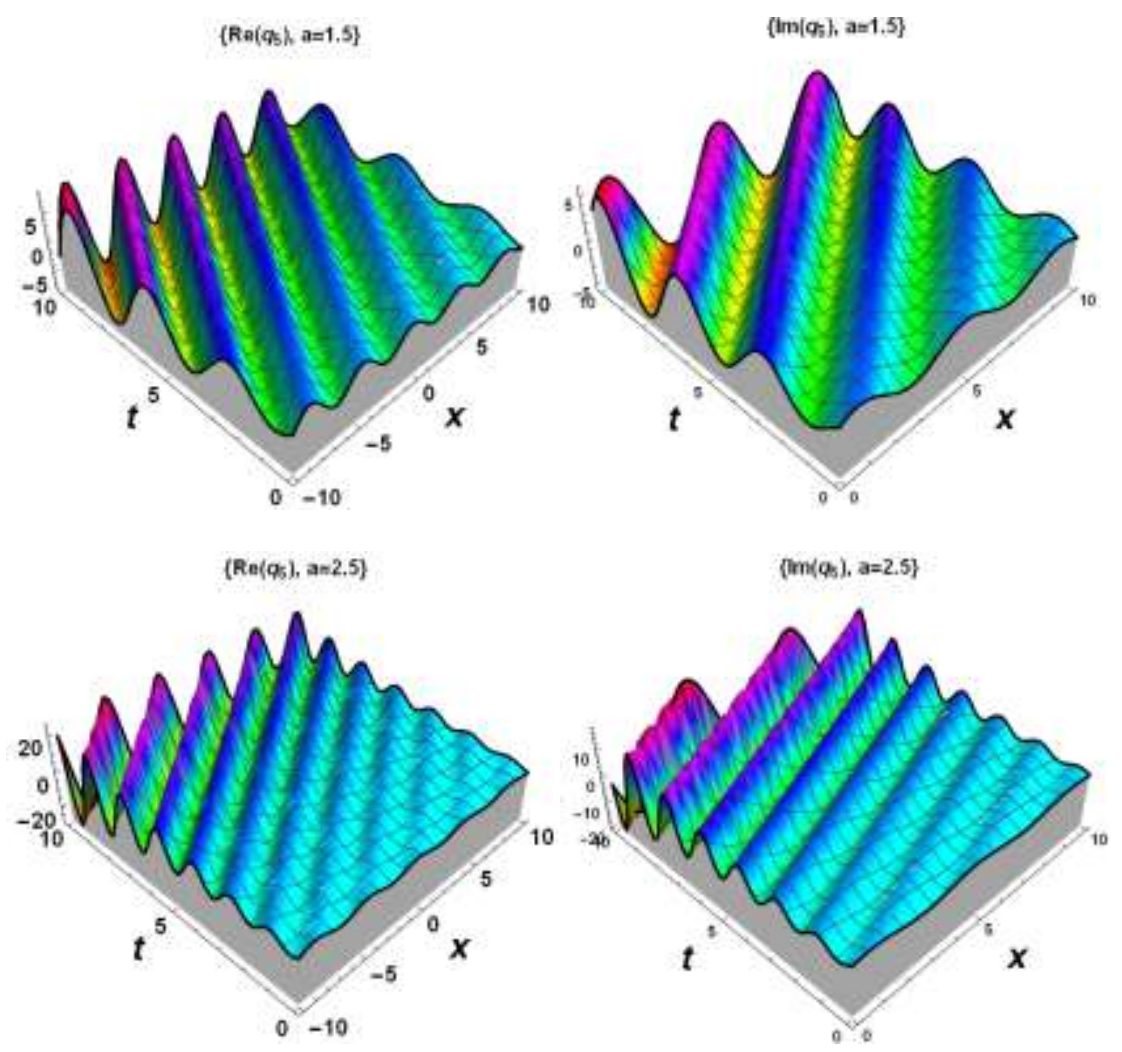

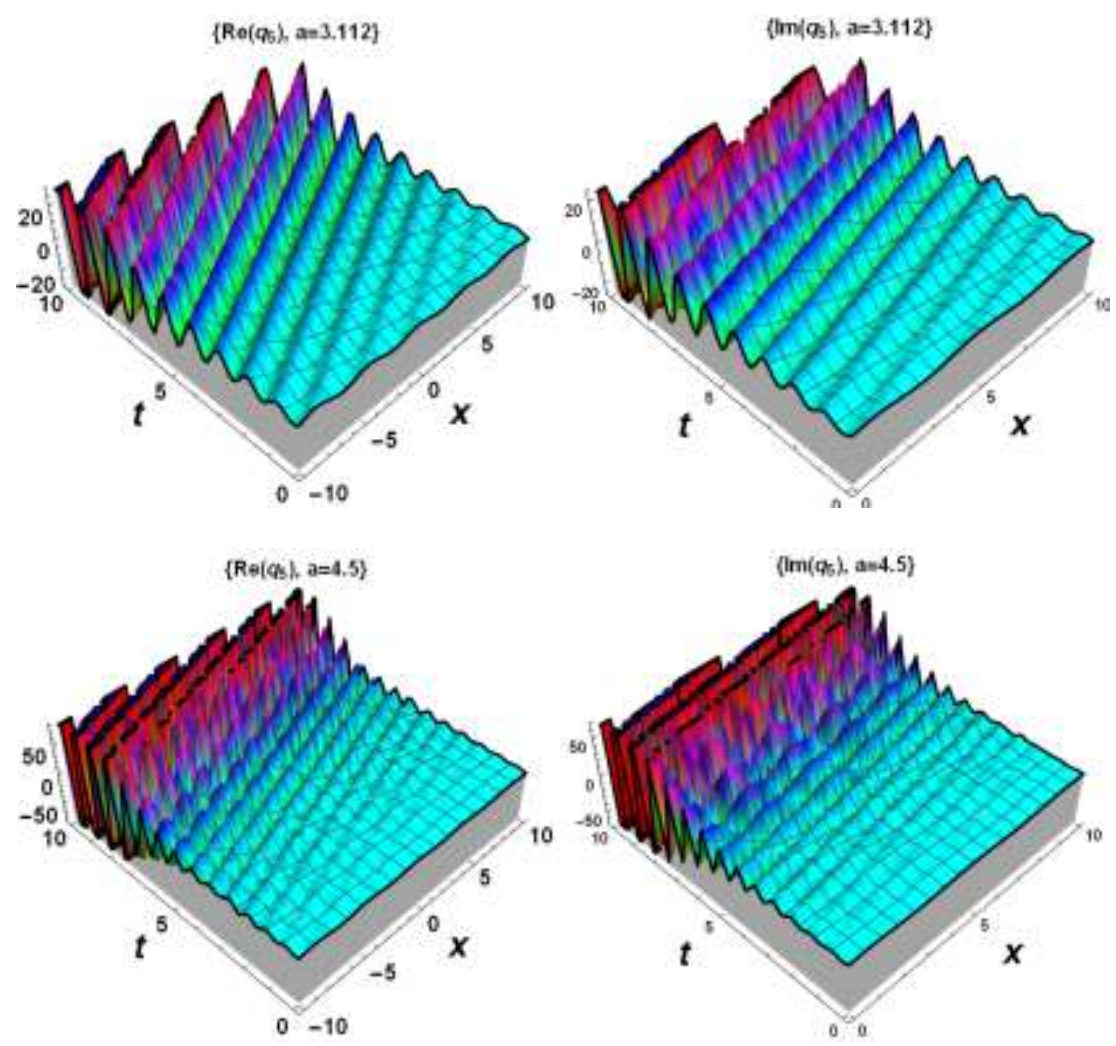

Fig.7: Simulation of $3 \mathrm{D}$ graphs of the Eq. (32) for $s=0.5, k=-1.5, \eta=0.4, \tau=0.1, \lambda=1, a_{0}=1, a_{2}=1, A=1.5, k=-1.5$.

As we observed in Fig. 7, the most important factor affecting the real part is the coefficient of the $q_{x x}$ term in the Eq. (1). It can be observed in Fig. 7 that as the coefficient " $a$ " increases, the wave's behavior deteriorates and refraction occurs for $a=3.112$. Depending on the velocity propagation parameter, while creating the simulation in Fig. 7, the parameters except the coefficient " $a$ " are taken as constant. Secondly, let's explain the behavior changes in the solitary wave solution within different values of the constant $\eta$, which is a variable of the wave frequency affecting the complex part, with the help of 3-dimensional simulation.
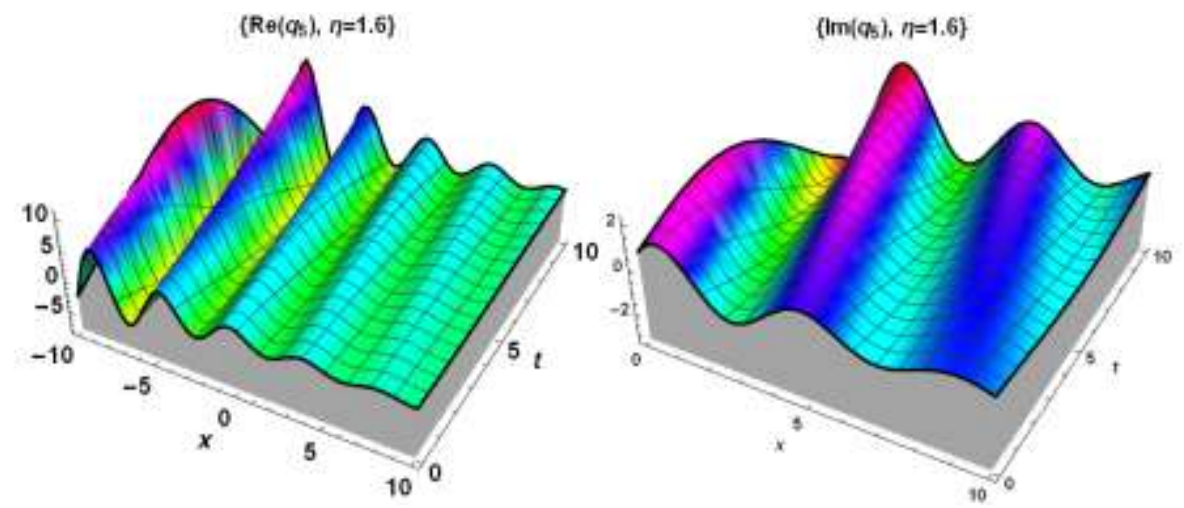

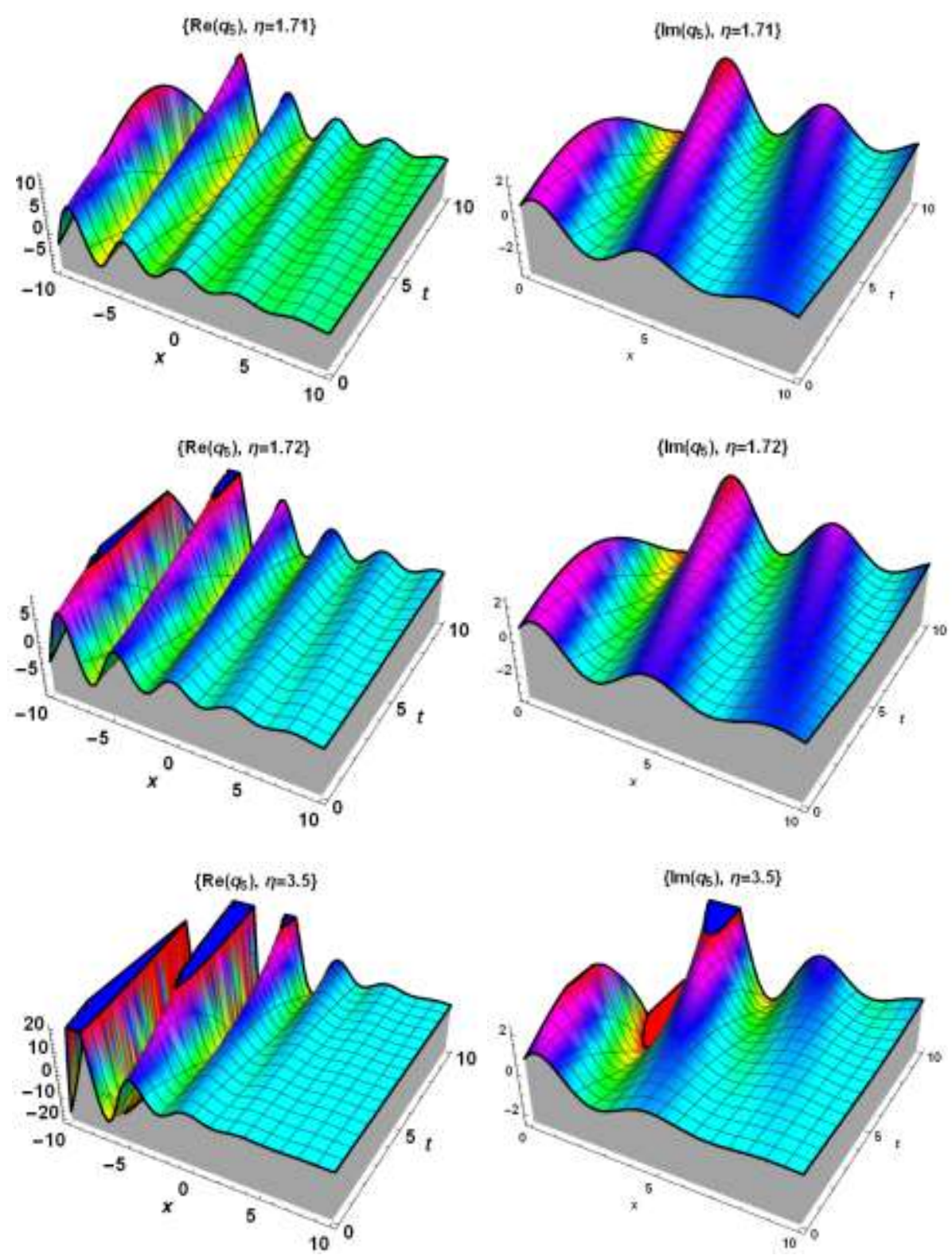

Fig.8: Simulation of 3D graphs of the Eq. (32) for $a=0.5, s=0.5, k=-1.5, \tau=0.1, \lambda=1, a_{0}=1, a_{2}=1, A=1.5$.

As we observed in Fig. 8, one of the most important factors affecting both the real and the imaginary part is that by increasing the expression of the wave frequency variable $\eta$, it is observed that disorders in wave behavior occur and physical refraction occurs at $\eta=1.72$ value. While creating the simulation in Fig. 8 that affects the frequency, the parameters except the coefficient $\eta$ are taken as constant. In this study, it has been verified that there are complex traveling wave solutions in the CLL equation, which is a nonlinear partial differential equation. After giving physical meanings to the coefficients in traveling wave solutions obtained in our study, these solutions can play a more important role in plasma and optical fiber.

The variation of the complex wave function for different values of the wave propagation $(\lambda)$ and velocity propagation parameters " $a$ " is shown in Figure 1. When Fig. 1 is examined, 
it can be seen that the value of " $b$ ", which is the nonlinear propagation parameter, is defined by the relationship between the nonlinear propagation coefficient $(\sigma)$ and self steeping parameter for short pulses $(\theta)$. In addition, the effects of changes in parameters and transformations in plasma and optical fiber were examined on figures. In this study, traveling wave solutions and interpretations are presented for $m=1$ density. In future studies, wave changes can be investigated for different values of intensity $\mathrm{m}$, which is a nonlinear parameter for complex wave function.

\section{Conclusions}

In this article, complex traveling wave solutions of the perturbed CLL equation are obtained by using modified $1 / G^{\prime}$-expansion and modified Kudryashov methods. Special solutions are obtained when the constants are matched with the real number in the solutions produced. These special solutions were presented with 3D, 2D and contour graphs. The advantages and disadvantages of both analytical methods are discussed. Besides, common and different aspects of both analytical methods were examined. In addition, a solitary wave was produced by giving special values to the parameters in the hyperbolic type complex traveling wave solution. Simulations for different values of the frequency and velocity propagation parameters of the single wave were presented in Figs. 7 and 8. For $a=3.112$ in Fig. 7 and for $\eta=1.72$ in Fig. 8, the values that cause break in the wave were calculated. Many complex operations such as drawing graphics and solving algebraic equation systems have been overcome by computer software programs. It was concluded that both analytical methods could be recommended for NLPDEs in the future.

\section{References}

[1] Durur, H. (2020). Different types analytic solutions of the (1+1)-dimensional resonant nonlinear Schrödinger's equation using $\left(\mathrm{G}^{\prime} / \mathrm{G}\right)$-expansion method. Modern Physics Letters B, 34(03), 2050036.

[2] Rehman, S. U., Yusuf, A., Bilal, M., Younas, U., Younis, M., \& Sulaiman, T. A. (2020). Application of $\left(\mathrm{G}^{\prime} / \mathrm{G}^{\wedge} 2\right)$-expansion method to microstructured solids, magneto-electro-elastic circular rod and (2+ 1)-dimensional nonlinear electrical lines. Journal| MESA, 11(4), 789-803.

[3] Durur, H., Yokuş, A., \& Kaya D., Hyperbolic Type Traveling Wave Solutions of Regularized Long Wave Equation. Bilecik Şeyh Edebali Üniversitesi Fen Bilimleri Dergisi, $7(2)$.

[4] Yokuş, A., Durur, H., \& Ahmad, H. (2020). Hyperbolic type solutions for the couple BoitiLeon-Pempinelli system. Facta Universitatis, Series: Mathematics and Informatics, 35(2), 523-531.

[5] Durur, H., \& Yokuş, A. Vakhnenko-Parkes Denkleminin Hiperbolik Tipte Yürüyen Dalga Çözümü. Erzincan Üniversitesi Fen Bilimleri Enstitüsü Dergisi, 13(2), 550-556.

[6] Yokuş, A., \& Durur, H. (2019). Complex hyperbolic traveling wave solutions of KuramotoSivashinsky equation using $\left(1 / \mathrm{G}^{\prime}\right)$ expansion method for nonlinear dynamic theory. Balikesir Üniversitesi Fen Bilimleri Enstitüsü Dergisi, 21(2), 590-599. 
[7] Durur, H., \& Yokuş, A. (2020). Analytical solutions of Kolmogorov-Petrovskii-Piskunov equation. Balıkesir Üniversitesi Fen Bilimleri Enstitüsü Dergisi, 22(2), 628-636.

[8] Ismael, H. F., Bulut, H., \& Baskonus, H. M. (2020). Optical soliton solutions to the FokasLenells equation via sine-Gordon expansion method and $\left(m+\left(\left\{G^{\prime}\right\} /\{G\}\right)\right)$-expansion method. Pramana, 94(1), 35.

[9] Durur, H., Ilhan, E., \& Bulut, H. (2020). Novel Complex Wave Solutions of the (2+1)Dimensional Hyperbolic Nonlinear Schrödinger Equation. Fractal and Fractional, 4(3), 41.

[10] Yokuş, A., Durur, H., Abro, K. A., \& Kaya, D. (2020). Role of Gilson-Pickering equation for the different types of soliton solutions: a nonlinear analysis. The European Physical Journal Plus, 135(8), 1-19.

[11] Yokus, A., Durur, H., Ahmad, H., Thounthong, P., \& Zhang, Y. F. (2020). Construction of exact traveling wave solutions of the Bogoyavlenskii equation by $\left(\mathrm{G}^{\prime} / \mathrm{G}, 1 / \mathrm{G}\right)$-expansion and $\left(1 / \mathrm{G}^{\prime}\right)$-expansion techniques. Results in Physics, 19, 103409.

[12] Yokus, A., Durur, H., Ahmad, H., \& Yao, S. W. (2020). Construction of Different Types Analytic Solutions for the Zhiber-Shabat Equation. Mathematics, 8(6), 908.

[13] Kayum, M. A., Barman, H. K., \& Akbar, M. A. Exact Soliton Solutions to the NanoBioscience and Biophysics Equations Through the Modified Simple Equation Method. In Proceedings of the Sixth International Conference on Mathematics and Computing (pp. 469482). Springer, Singapore.

[14] Seadawy, A. R., Lu, D., \& Khater, M. M. (2017). Bifurcations of traveling wave solutions for Dodd-Bullough-Mikhailov equation and coupled Higgs equation and their applications. Chinese journal of physics, 55(4), 1310-1318.

[15] Chen, L., Yang, L., Zhang, R., \& Cui, J. (2019). Generalized (2+ 1)-dimensional mKdVBurgers equation and its solution by modified hyperbolic function expansion method. Results in Physics, 13, 102280.

[16] Gu, Y., Yuan, W., Aminakbari, N., \& Jiang, Q. (2017). Exact solutions of the VakhnenkoParkes equation with complex method. Journal of Function Spaces, 2017.

[17] Pervaiz, N., \& Aziz, I. (2020). Haar wavelet approximation for the solution of cubic nonlinear Schrodinger equations. Physica A: Statistical Mechanics and its Applications, 545, 123738.

[18] Kirs, M., Karjust, K., Aziz, I., Õunapuu, E., \& Tungel, E. (2018). Free vibration analysis of a functionally graded material beam: evaluation of the Haar wavelet method. Proceedings of the Estonian Academy of Sciences, 67(1).

[19] Durur, H., Tasbozan, O., \& Kurt, A. (2020). New Analytical Solutions of Conformable Time Fractional Bad and Good Modified Boussinesq Equations. Applied Mathematics and Nonlinear Sciences, 5(1), 447-454. 
[20] Durur, H., Kurt, A., \& Tasbozan, O. (2020). New Travelling Wave Solutions for KdV6 Equation Using Sub Equation Method. Applied Mathematics and Nonlinear Sciences, 5(1), 455-460.

[21] Guerrero Sánchez, Y., Sabir, Z., Günerhan, H., \& Baskonus, H. M. (2020). Analytical and approximate solutions of a novel nervous stomach mathematical model. Discrete Dynamics in Nature and Society, 2020.

[22] Yokus, A., Kuzu, B., \& Demiroğlu, U. (2019). Investigation of solitary wave solutions for the (3+1)-dimensional Zakharov-Kuznetsov equation. International Journal of Modern Physics B, 33(29), 1950350.

[23] Kudryashov, N. A. (2020). Optical solitons of mathematical model with arbitrary refractive index. Optik, 224, 165391.

[24] Yavuz, M., \& Yokus, A. (2020). Analytical and numerical approaches to nerve impulse model of fractional-order. Numerical Methods for Partial Differential Equations, 36(6), 13481368 .

[25] Yokus, A., Kuzu, B., \& Demiroğlu, U. (2019). Investigation of solitary wave solutions for the (3+1)-dimensional Zakharov-Kuznetsov equation. International Journal of Modern Physics B, 33(29), 1950350.

[26] Kudryashov, N. A. (2020). On traveling wave solutions of the Kundu-Eckhaus equation. Optik, 224, 165500.

[27] Yokuş, A., \& Kaya, D. (2020). Comparison exact and numerical simulation of the traveling wave solution in nonlinear dynamics. International Journal of Modern Physics B, 2050282.

[28] Kudryashov, N. A. (2020). Periodic and solitary waves in optical fiber Bragg gratings with dispersive reflectivity. Chinese Journal of Physics, 66, 401-405.

[29] Modanli, M. (2019). On the numerical solution for third order fractional partial differential equation by difference scheme method. An International Journal of Optimization and Control: Theories \& Applications (IJOCTA), 9(3), 1-5.

[30] Tala-Tebue, E., Tetchoka-Manemo, C., Rezazadeh, H., Bekir, A., \& Chu, Y. M. (2020). Optical solutions of the (2+1)-dimensional hyperbolic nonlinear Schrödinger equation using two different methods. Results in Physics, 19, 103514.

[31] Vahidi, J., Zabihi, A., Rezazadeh, H., \& Ansari, R. (2020). New extended direct algebraic method for the resonant nonlinear Schrödinger equation with Kerr law nonlinearity. Optik, 165936.

[32] Triki, Houria, et al. "Chirped w-shaped optical solitons of Chen-Lee-Liu equation." Optik 155 (2018): 208-212.

[33] Chen, H. H., Y. C. Lee, and C. S. Liu. "Integrability of nonlinear Hamiltonian systems by inverse scattering method." Physica Scripta 20.3-4 (1979): 490.

[34] Yıldirım, Yakup, et al. "Optical soliton perturbation with Chen-Lee-Liu equation." Optik 220 (2020): 165177. 
[35] Triki, Houria, M. M. Babatin, and Anjan Biswas. "Chirped bright solitons for Chen-LeeLiu equation in optical fibers and PCF." Optik 149 (2017): 300-303.

[36] Triki, Houria, et al. "Chirped dark and gray solitons for Chen-Lee-Liu equation in optical fibers and PCF." Optik 155 (2018): 329-333.

[37] Biswas, Anjan, et al. "Chirped optical solitons of Chen-Lee-Liu equation by extended trial equation scheme." Optik 156 (2018): 999-1006.

[38] Yıldırım, Y., Biswas, A., Asma, M., Ekici, M., Ntsime, B. P., Zayed, E. M. E., ... \& Belic, M. R. (2020). Optical soliton perturbation with Chen-Lee-Liu equation. Optik, 220, 165177. 

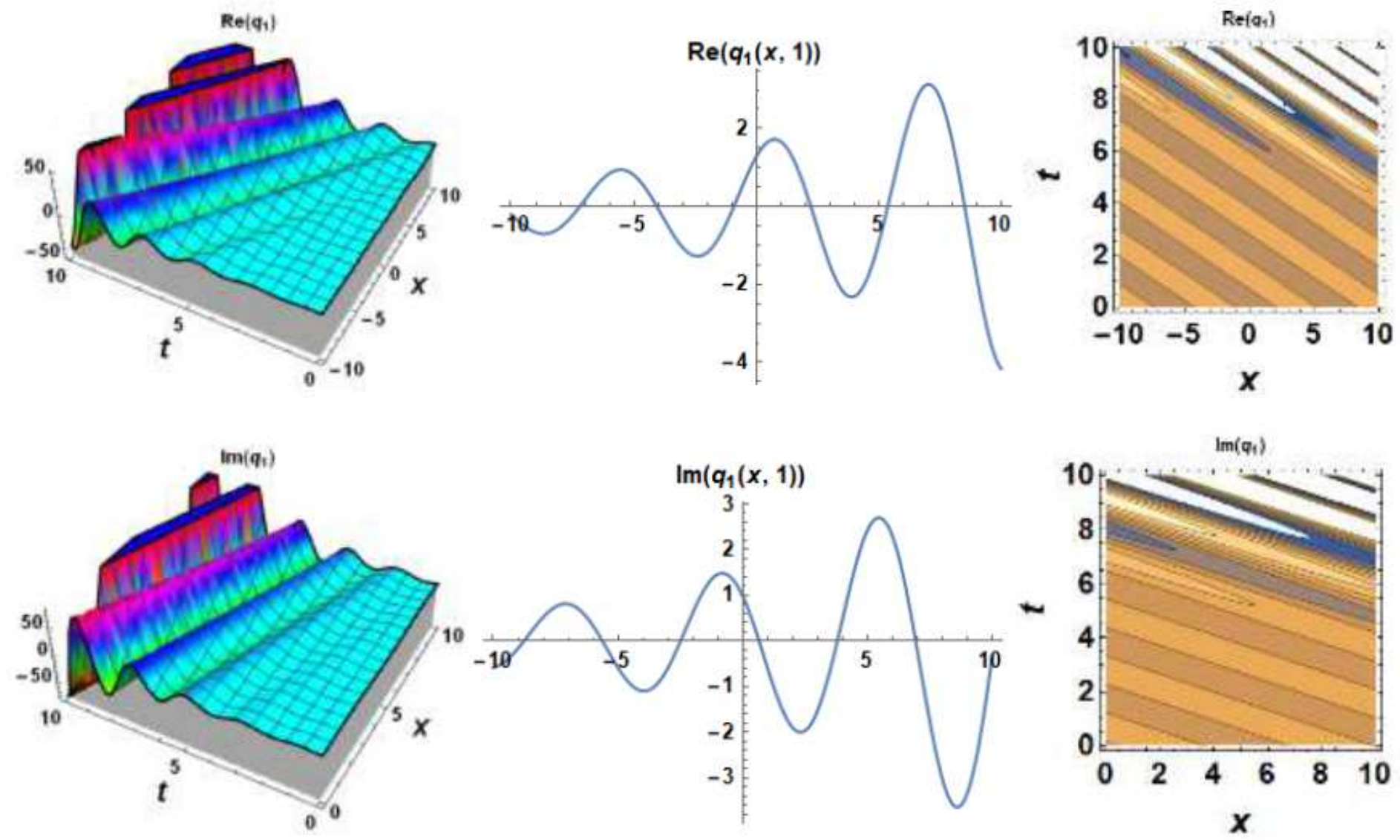

Figure 1

Real and imaginary parts of 3D, 2D and contour graphs for the Eq. (21) see manuscript .pdf for full figure caption. 

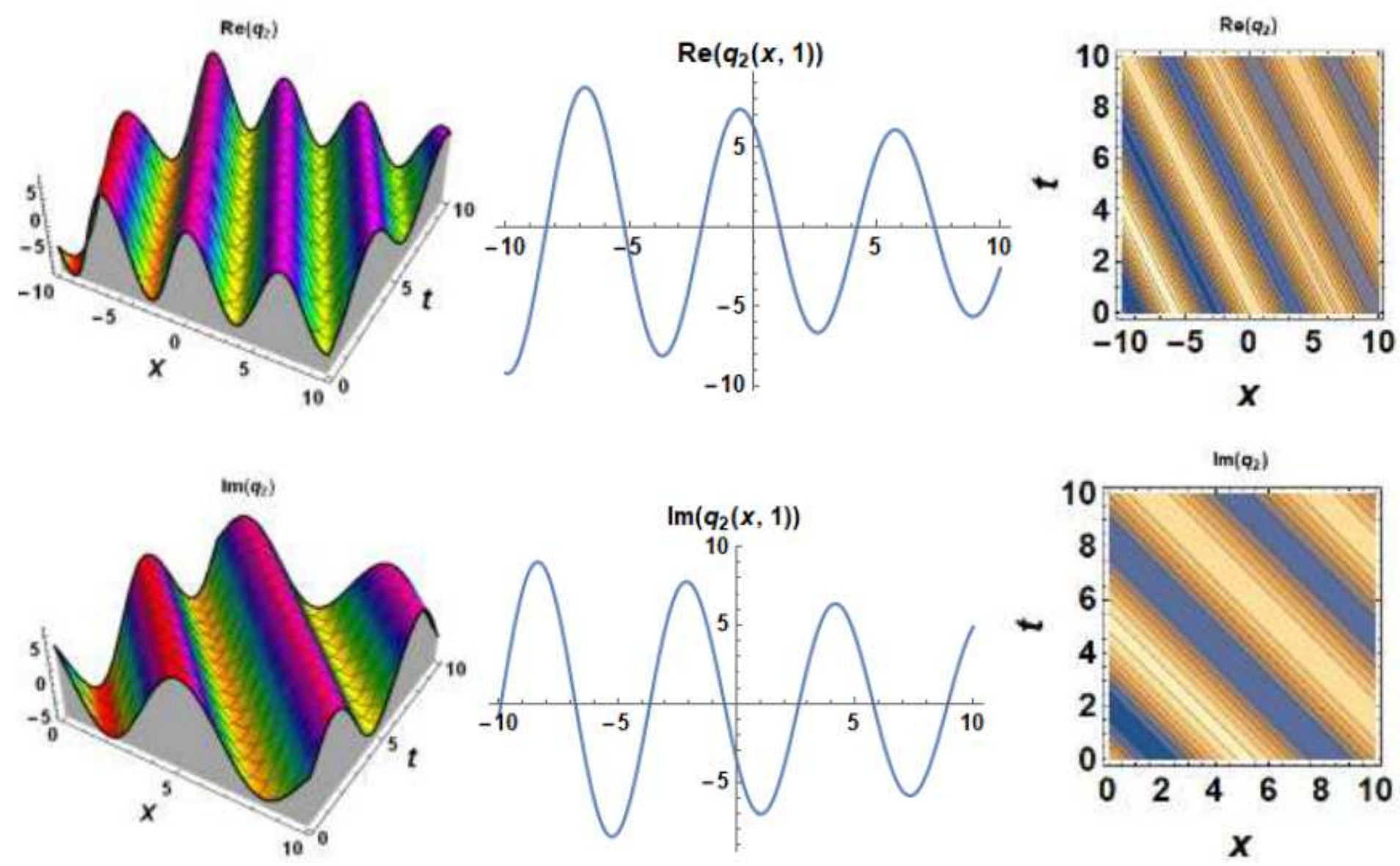

Figure 2

Real and imaginary parts of 3D, 2D and contour graphs of the Eq. (24) see manuscript .pdf for full figure caption. 

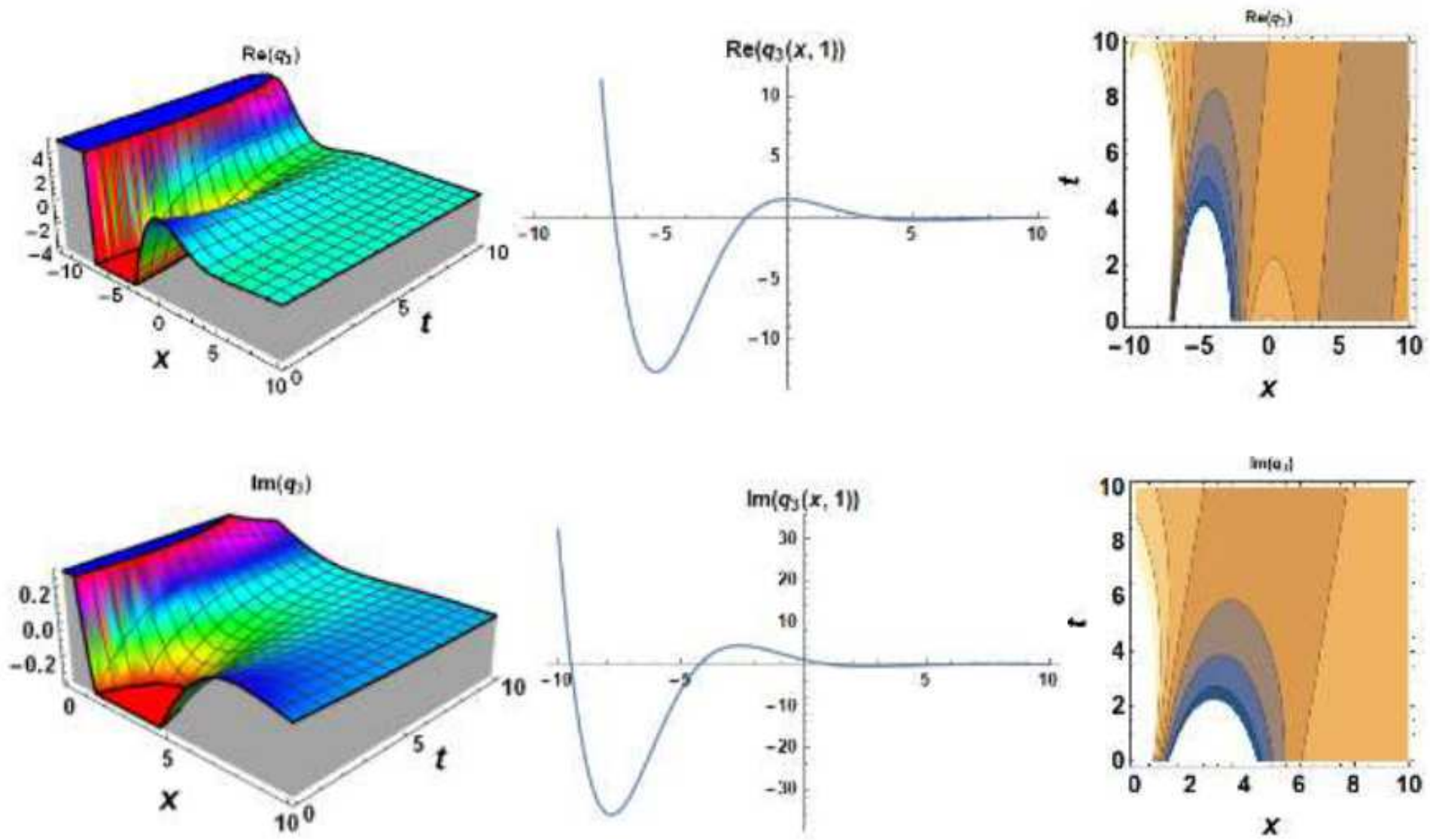

Figure 3

Real and imaginary parts of 3D, 2D and contour graphs of the Eq. (28) see manuscript .pdf for full figure caption. 

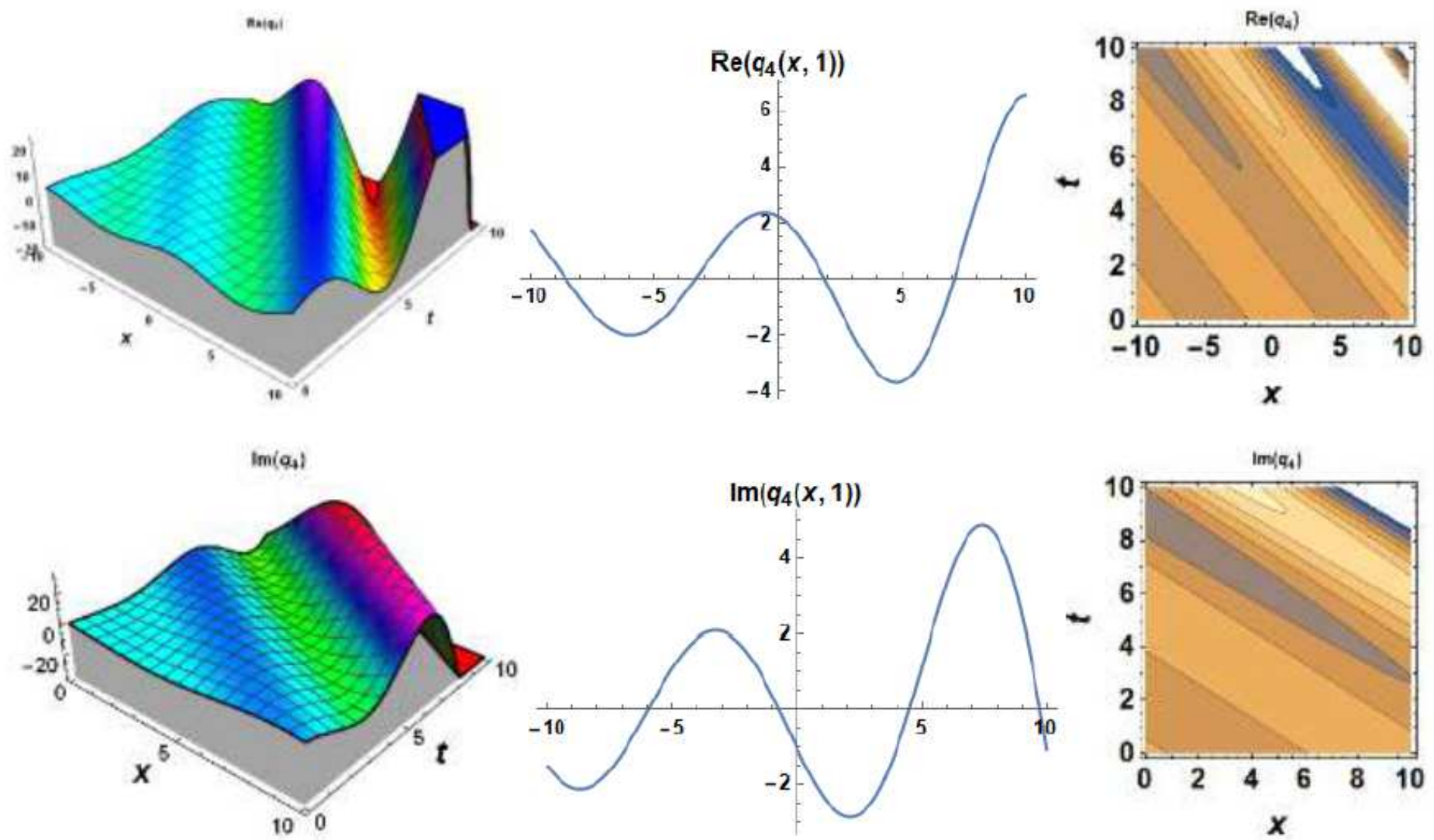

Figure 4

Real and imaginary parts of 3D, 2D and contour graphs of the Eq. (28) see manuscript .pdf for full figure caption. 

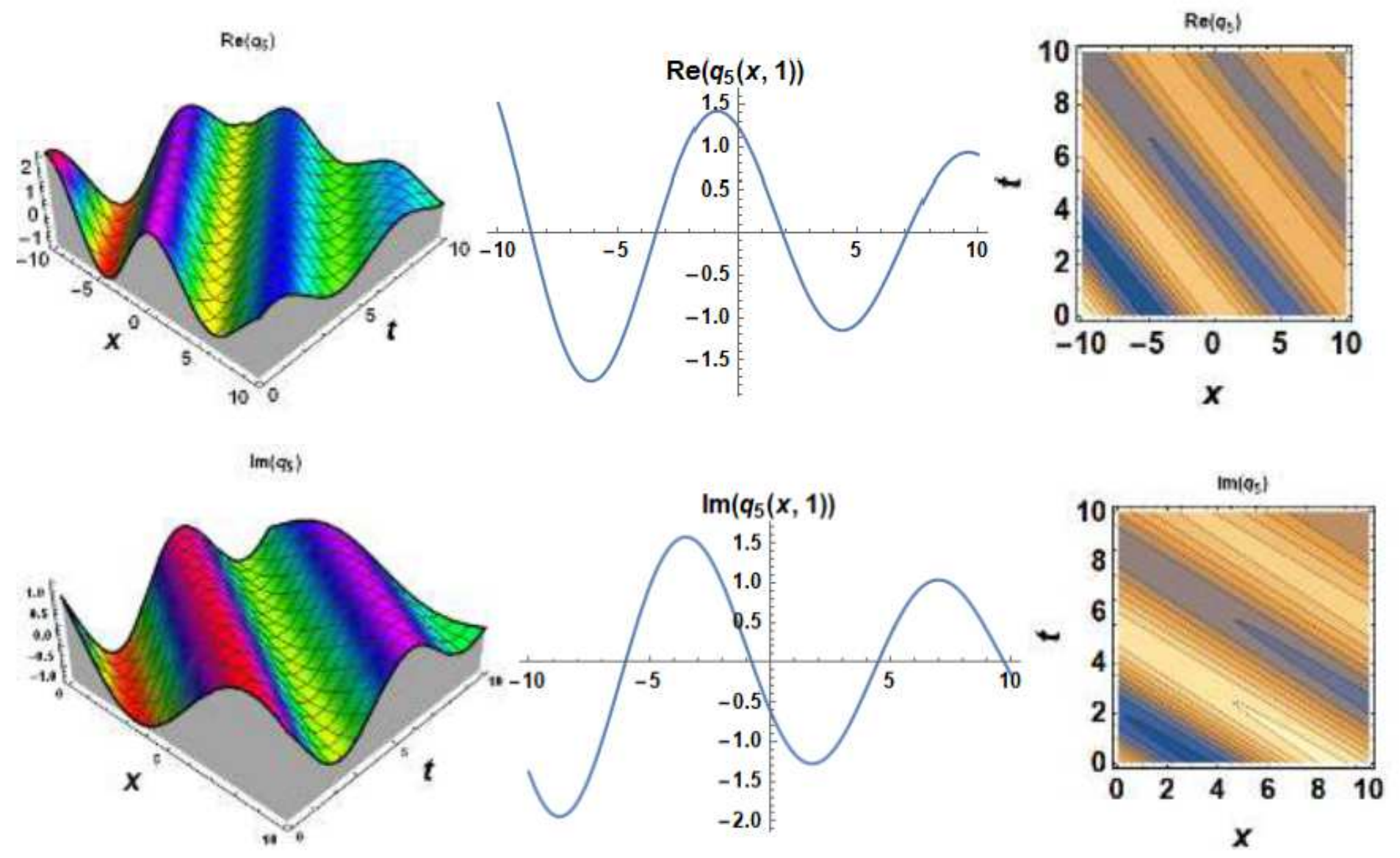

Figure 5

Real and imaginary of 3D, 2D and contour graphs of the Eq. (32) see manuscript .pdf for full figure caption. 

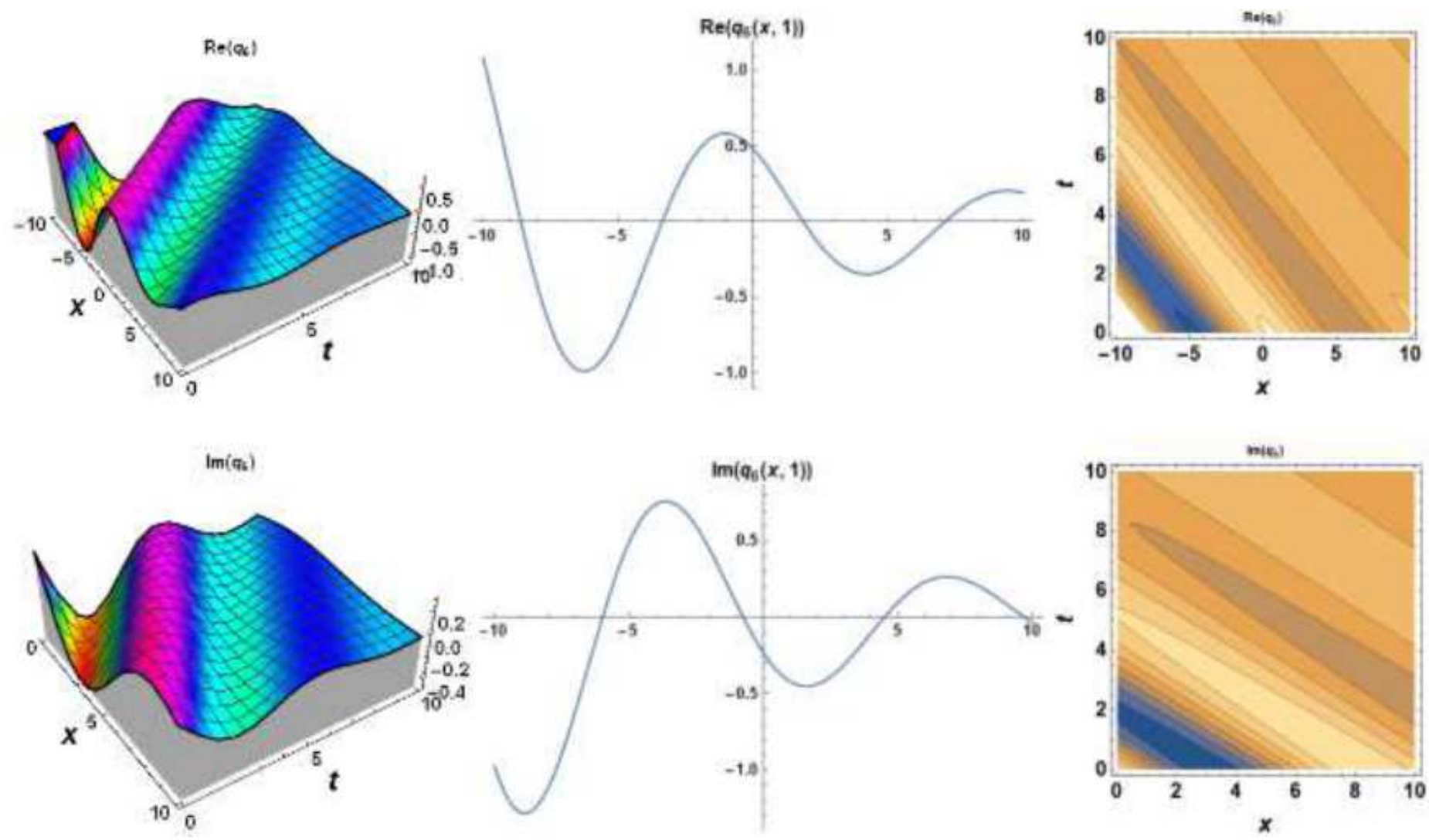

Figure 6

Real and imaginary of 3D, 2D and contour graphs of the Eq. (34) see manuscript .pdf for full figure caption.
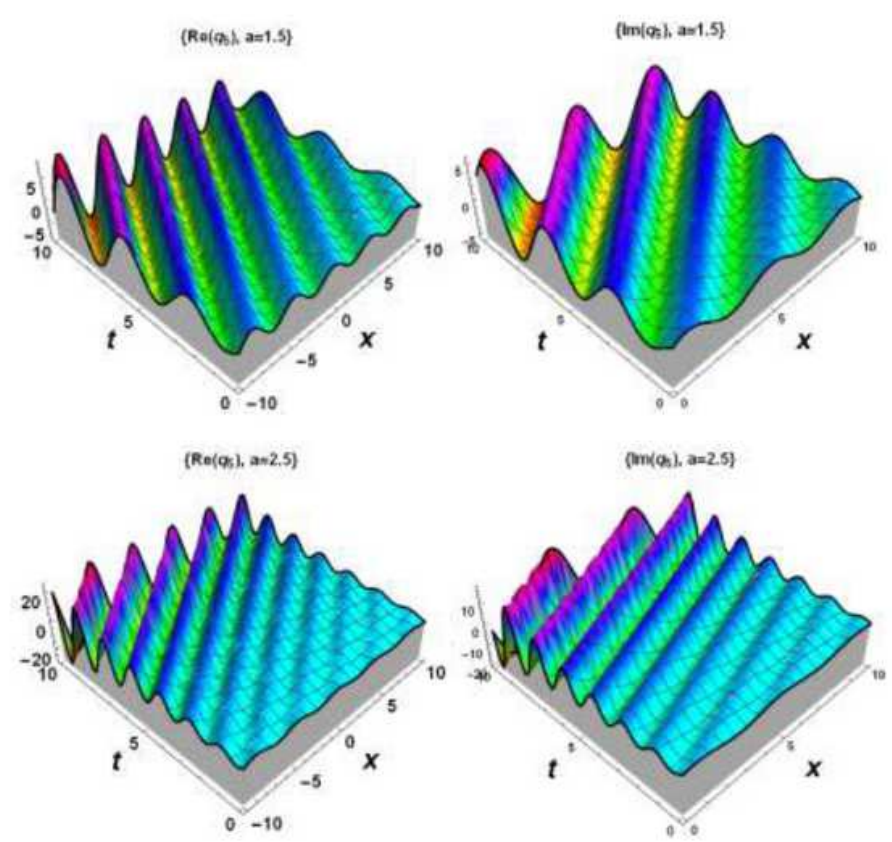
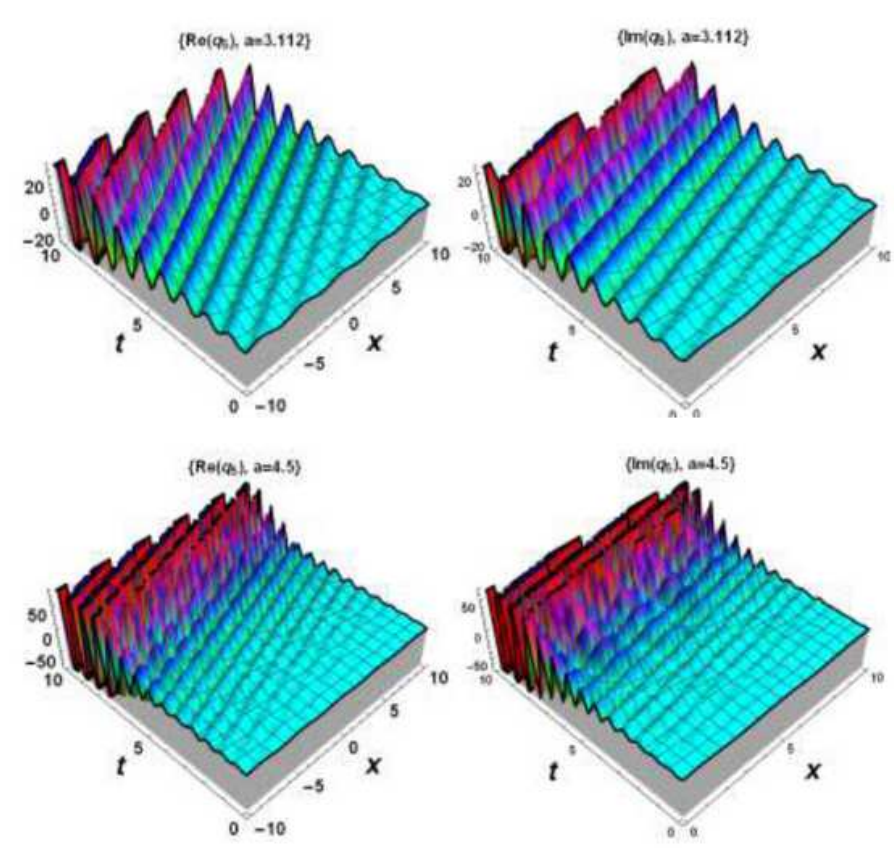

Figure 7 
Simulation of 3D graphs of the Eq. (32) see manuscript .pdf for full figure caption.
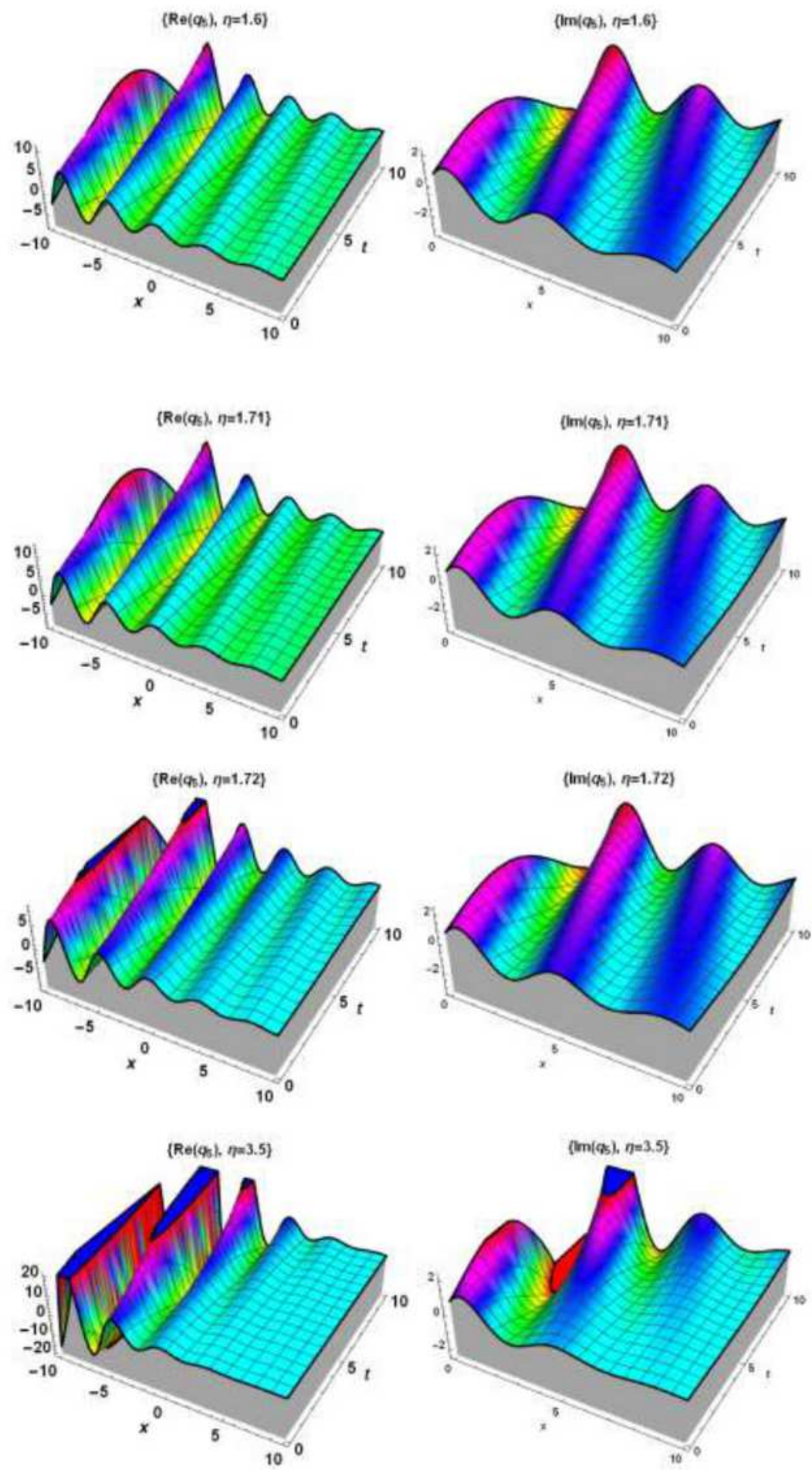

Figure 8

Simulation of 3D graphs of the Eq. (32) see manuscript .pdf for full figure caption. 